\title{
Rat Claustrum Coordinates But Does Not Integrate Somatosensory and Motor Cortical Information
}

\author{
Jared B. Smith, Harsha Radhakrishnan, and Kevin D. Alloway \\ Department of Neural and Behavioral Sciences, Center for Neural Engineering, Pennsylvania State University, University Park, Pennsylvania 16802
}

The function of the claustrum is a fundamental issue in neuroscience. Anatomical data indicate that the rat claustrum is part of an interhemispheric circuit that could be involved in the bilateral coordination of whisker movements. Given that whisking is a somestheticguided motor behavior, the goal of the current study was to elucidate the connections of the claustrum with respect to the whisker representations in the primary somatosensory (wSI) and motor (wMI) cortical areas. Anterograde tracer injections showed that wMI projects most densely to the claustrum in the contralateral hemisphere, whereas wSI does not project to the claustrum in either hemisphere. Injections of different retrograde tracers into wMI and wSI of the same animal revealed intermingled populations of labeled neurons in the claustrum, as well as many double-labeled neurons. This indicates that the same part of the claustrum projects to the whisker representations in both SI and MI. Finally, injections of different anterograde tracers in the wMI regions of both hemispheres were combined with a retrograde tracer injection in wSI, and this produced dense terminal labeling around retrogradely labeled neurons in the claustrum of both hemispheres. Although the rodent claustrum is probably involved in the interhemispheric coordination of the MI and SI whisker representations, it does not receive inputs from both of these cortical regions. Hence, the claustrum should not be universally regarded as an integrator of somesthetic and motor information.

\section{Introduction}

Due to its connections with many parts of neocortex, the claustrum has been portrayed as a global integrator of cortical information (Edelstein and Denaro, 2004; Crick and Koch, 2005). This view is based largely on studies in which injections of a single anterograde tracer in different animals indicate that many cortical areas project to the claustrum (Olson and Graybiel, 1980; Pearson et al., 1982; Sherk, 1986). In these studies, similar parts of the claustrum appear to receive projections from separate cortical regions having different sensory or motor functions, but no study has administered different anterograde tracers into separate cortical regions of the same animal to demonstrate converging corticoclaustral projections. Furthermore, the role of the claustrum as an integrator of multimodal information has recently been questioned by physiological data showing that claustral neurons respond to a single sensory modality but not to multimodal sensory inputs (Remedios et al., 2010).

Our anatomical work in rats has revealed an interhemispheric cortico-claustro-cortical pathway that supplements the direct callosal interconnections between the motor whisker representations in the two hemispheres (Alloway et al., 2009; Smith and Alloway, 2010). This finding is functionally significant because rodent whisking behavior displays a great deal of bilateral coor-

\footnotetext{
Received March 28, 2012; revised May 7, 2012; accepted May 11, 2012.

Author contributions: J.B.S. and K.D.A. designed research; J.B.S. and H.R. performed research; J.B.S., H.R., and K.D.A. analyzed data; J.B.S. and K.D.A. wrote the paper.

This work was supported by NIH Grant NS37532.

Correspondence should be addressed to Dr. Kevin D. Alloway, Center for Neural Engineering, Millennium Science Complex W-316, Pennsylvania State University, University Park, PA 16802. E-mail: kda1@psu.edu.

DOI:10.1523/JNEUROSCI.1524-12.2012

Copyright $\odot 2012$ the authors $\quad 0270-6474 / 12 / 328583-06 \$ 15.00 / 0$
}

dination (Towal and Hartmann, 2006; Mitchinson et al., 2007). In cats, microstimulation of the claustrum exerts bilateral influences on the motor cortical neurons that control eye movements (Cortimiglia et al., 1991). Together, these findings suggest that the claustrum is important for regulating the bilateral coordination of certain behavioral movements. Consistent with this view, the claustrum is connected with the motor areas in both hemispheres (Li et al., 1986; Sloniewski et al., 1986), and one study has reported that some claustral neurons have widely divergent axonal collaterals that allow them to innervate motor regions in each hemisphere (Minciacchi et al., 1985).

Rodent tracing studies indicate some overlap among populations of claustral neurons that project to the somatosensory and motor cortical areas, but claustral neurons that project to both primary somatosensory (SI) and motor (MI) cortices have not been observed (Li et al., 1986; Sloniewski et al., 1986). These previous retrograde tracing studies, however, relied exclusively on stereotaxic coordinates to locate injection sites in SI and MI. Consequently, because physiology-based mapping techniques were not used to determine tracer placement, it is possible that corresponding representations in SI and MI were not injected.

To characterize the sensorimotor input-output connections of the claustrum, we injected different combinations of anterograde and retrograde tracers in physiologically defined sites in the SI and MI whisker representations of rats (wSI and wMI, respectively). Paired injections of different anterograde tracers in these cortical regions indicate that the claustrum receives inputs from wMI, especially from the contralateral hemisphere, but not from the wSI region of either hemisphere. Paired injections of different retrograde tracers revealed overlapping retrograde labeling in the claustrum of each hemisphere with many double-labeled neu- 
rons. When anterograde tracer deposits in wMI of both hemispheres were coupled with a retrograde tracer deposit in wSI, corticoclaustral projections from wMI terminated densely around claustral neurons that project to wSI. These data indicate that the rat claustrum transmits information to both SI and MI, but it does not integrate information from both of these regions.

\section{Materials and Methods}

Anatomical tracer injections were made in adult male Sprague Dawley rats (Charles River) weighing 300-800 g. All procedures conformed to $\mathrm{NIH}$ guidelines and were approved by our Institutional Animal Care and Use Committee.

Animal surgery. Rats were initially sedated with an intramuscular injection of ketamine $\mathrm{HCl}(40 \mathrm{mg} / \mathrm{kg})$ and xylazine $(12 \mathrm{mg} / \mathrm{kg})$, and then received intramuscular injections of atropine methyl nitrate $(0.5 \mathrm{mg} / \mathrm{kg})$, dexamethasone sodium phosphate $(5 \mathrm{mg} / \mathrm{kg})$, and chloramphenicol (50 $\mathrm{mg} / \mathrm{kg}$ ). Each rat was intubated, placed in a stereotaxic frame, and ventilated with oxygen. A homeothermic heating blanket maintained body temperature at $37.0^{\circ} \mathrm{C}$, and ophthalmic ointment was applied to avoid corneal drying. Heart rate, arterial blood oxygen saturation, and endtidal carbon dioxide were monitored throughout each surgery. A midline incision was made over the cranium, and lidocaine (2\%) was injected into the wound margins. A hole was drilled over the cerebellum to allow insertion of a ground screw. Craniotomies were made over the somatosensory (1-4 mm caudal, 3-7 mm lateral to bregma) and motor (1-3 mm rostral, $0.5-3 \mathrm{~mm}$ lateral to bregma) cortical areas based on previous reports (Hall and Lindholm, 1974; Hoffer et al., 2003; Brecht et al., 2004; Haiss and Schwarz, 2005).

Tracer injections. Rats received combined injections of different anterograde and retrograde tracers in physiologically defined cortical regions (i.e., wSI and wMI). The first set of rats received paired injections of anterograde tracers, namely 15\% Fluororuby (FR; D-1817; Invitrogen) and 15\% biotinylated dextran amine (BDA; D-7135; Invitrogen). The second set of rats received paired injections of retrograde tracers, namely $2 \%$ True Blue (TB; T-1323; Invitrogen) and 2\% Fluorogold (FG; $\mathrm{H}-22845$; Fluoro-Chrome). The final group of rats received two anterograde tracer injections, 15\% FR in the left wMI and 15\% Alexa Fluor 488 (AF; D-2290; Invitrogen) in the right wMI, and an injection of a retrograde tracer, $2 \% \mathrm{FG}$, in the wSI of the right hemisphere.

Most tracers (BDA, FR, AF, and TB) were pressure injected via Hamilton syringes with pulled glass pipettes $(\sim 60 \mu \mathrm{m}$ tip diameter) cemented on the tip of the syringe needle. A volume of $75 \mathrm{nl}$ was injected at each of two depths (1.7 and $1.0 \mathrm{~mm}$ below pial surface). Deposits of FG were iontophoretically injected using positive current pulses at $2-5 \mu \mathrm{A}$ for $10-20 \mathrm{~min}$ (with a $7 \mathrm{~s}$ on/off duty cycle) at multiple depths (1.6, 1.0, and $0.4 \mathrm{~mm}$ below the pia).

To identify wMI, a pipette filled with $3 \mathrm{M} \mathrm{NaCl}(0.5-1.0 \mathrm{M} \Omega)$ was inserted orthogonal to the pial surface to a depth corresponding to layer $\mathrm{V}(\sim 1.5 \mathrm{~mm}$ deep $)$. Low amplitude current $(<50 \mu \mathrm{A})$ was administered as $0.7 \mathrm{~ms}$ pulses at a rate of $250 \mathrm{~Hz}$ for an $80 \mathrm{~ms}$ period to elicit muscle twitches. The stimulating electrode was moved to map the extent of wMI (both retraction and rhythmic whisking zones) relative to other representations including forelimb, neck, and nose representations, as described in previous reports (Sanderson et al., 1984; Brecht et al., 2004; Haiss and Schwarz, 2005; Smith and Alloway, 2010). After removing the stimulating electrode, tracers were injected at multiple sites within the wMI retraction zone, but were at least $500 \mu \mathrm{m}$ from other representations. Although the retraction and rhythmic whisking zones project to overlapping parts of the claustrum (Smith and Alloway, 2010), we injected the retraction zone because this part of $\mathrm{wMI}$ is closest to the MI area that receives direct projections from SI barrel cortex (J. B. Smith and K. D. Alloway, unpublished data).

Injection sites in wSI were determined by receptive field mapping using a saline-filled pipette. The pipette was tilted at an angle of $25^{\circ}$ to the sagittal plane and stereotaxically positioned above regions known to correspond to wSI (Hall and Lindholm, 1974; Hoffer et al., 2003). A dural slit was made and the pipette was lowered 400-800 $\mu \mathrm{m}$ below the pial surface, corresponding to cortical layer IV. A silver wire with a male gold pin was placed in the pipette and connected to a cable attached to the headstage of the amplifier. Extracellular potentials were bandpass filtered $(300-3000 \mathrm{~Hz})$ and amplified with a Dagan amplifier (Model 2200). Neuronal activity was visualized on a digital oscilloscope (DPO4034; Tektronix) and played through an audio monitor. Neuronal receptive fields were identified by manual deflection of individual whiskers with a wooden probe. Multiple sites in wSI were mapped and injected so that the tracers would fill SI barrel cortex.

Histology. Following tracer injections, wound margins were sutured and rats were returned to their home cage for 7-10 $\mathrm{d}$ to permit tracer transport. Subsequently, rats were deeply sedated with intramuscular injections of ketamine $(60 \mathrm{mg} / \mathrm{kg})$ and xylazine $(18 \mathrm{mg} / \mathrm{kg})$, and were transcardially perfused with heparinized saline, $4 \%$ paraformaldehyde, and $4 \%$ paraformaldehyde with $10 \%$ sucrose. Brains were removed and refrigerated overnight in $4 \%$ paraformaldehyde with $30 \%$ sucrose. Olfactory bulbs and the hindbrain were removed and a slit was made ventrally in the left hemisphere to orient sections for mounting. Brains were cut into $60 \mu \mathrm{m}$ serially ordered sections by a freezing microtome and stored in $0.1 \mathrm{~m}$ PBS. Sections were divided into three series. The first series was mounted onto gelatin-coated slides and dried overnight before staining with thionin. The second series was processed to visualize tracer labeling. Brains containing only fluorescent tracers were mounted and dried overnight before dehydrating in ethanol, defatting in xylene, and coverslipping with Cytoseal. For cases injected with BDA, sections were processed to visualize the tracer using heavy metal-enhanced peroxidase immunohistochemisry as previously described (Kincaid and Wilson, 1996; Alloway et al., 1998). Briefly, sections were agitated in $0.3 \% \mathrm{H}_{2} \mathrm{O}_{2}$ and then $0.3 \%$ Triton X-100, $0.1 \mathrm{M}$ PBS before being incubated for $2-4 \mathrm{~h}$ in activated avidin-biotinylated horseradish peroxidase solution (Vector Novocostra Laboratories). After rinses in $0.1 \mathrm{M}$ PBS, sections were incubated in $0.05 \% \mathrm{DAB}, 0.0005 \% \mathrm{H}_{2} \mathrm{O}_{2}, 0.04 \% \mathrm{NiCl} 2$ in $0.1 \mathrm{M}$ tris buffer, $\mathrm{pH} 7.2$, for $9-12 \mathrm{~min}$. The reaction was then stopped by subsequent washes in 0.1 M PBS. Following immunohistochemical processing to reveal BDA labeling, sections were mounted, dried overnight, and coverslipped. The third series was processed for cytochrome oxidase (Land and Simons, 1985).

Anatomical analysis. Tracer labeling was analyzed with brightfield and fluorescent microscopy using an Olympus BH-2 microscope. Photomicrographs were obtained with a Retiga EX CCD digital camera (Qimaging) mounted on the microscope. One UV filter was used to visualize TB and FG (11000v2; Chroma Technologies), and a combined FITC/TRITC filter (51004v2; Chroma Technologies) was used to visualize FR and AF labeling. Brightfield microscopy was used to see BDA labeling. Digital reconstructions of tracer labeling relative to anatomical landmarks were made using an MDPlot system (Accustage) interfaced with the microscope. Retrogradely labeled neurons were required to display at least one prominent dendritic process to be plotted. Anterogradely labeled beaded varicosities (en passant and terminal) were plotted to indicate putative synapses (Voigt et al., 1993; Kincaid and Wilson, 1996).

Some images were captured by a laser confocal microscope (TCS SP2 AOBS; Leica Microsystems) with a $63 \times$ oil-immersion objective. Each tracer was scanned separately at specific excitation/detection specifications per optical slice, and maximum projection composites were constructed after imaging. In dual retrograde cases, TB (405 nm excitation, 410-460 nm detection) and FG (405 nm excitation, 520-600 nm detection) were imaged to reveal double-labeled neurons. Cases that received three tracer injections were visualized for AF-labeling (488 $\mathrm{nm}$ excitation, 500-565 nm detection), FR-labeling ( $543 \mathrm{~nm}$ excitation, 565-630 $\mathrm{nm}$ detection), and FG-labeled neurons (as described above).

\section{Results}

Connections between wMI, wSI, and the claustrum were analyzed in three sets of experiments. Paired injections of the anterograde tracers FR and BDA in wMI and wSI $(n=3)$ were performed to determine whether these sensorimotor cortical regions project to overlapping parts of the claustrum. Paired injections of retrograde tracers TB and FG in wMI and wSI $(n=3)$ were made to determine whether the same part of the claustrum 


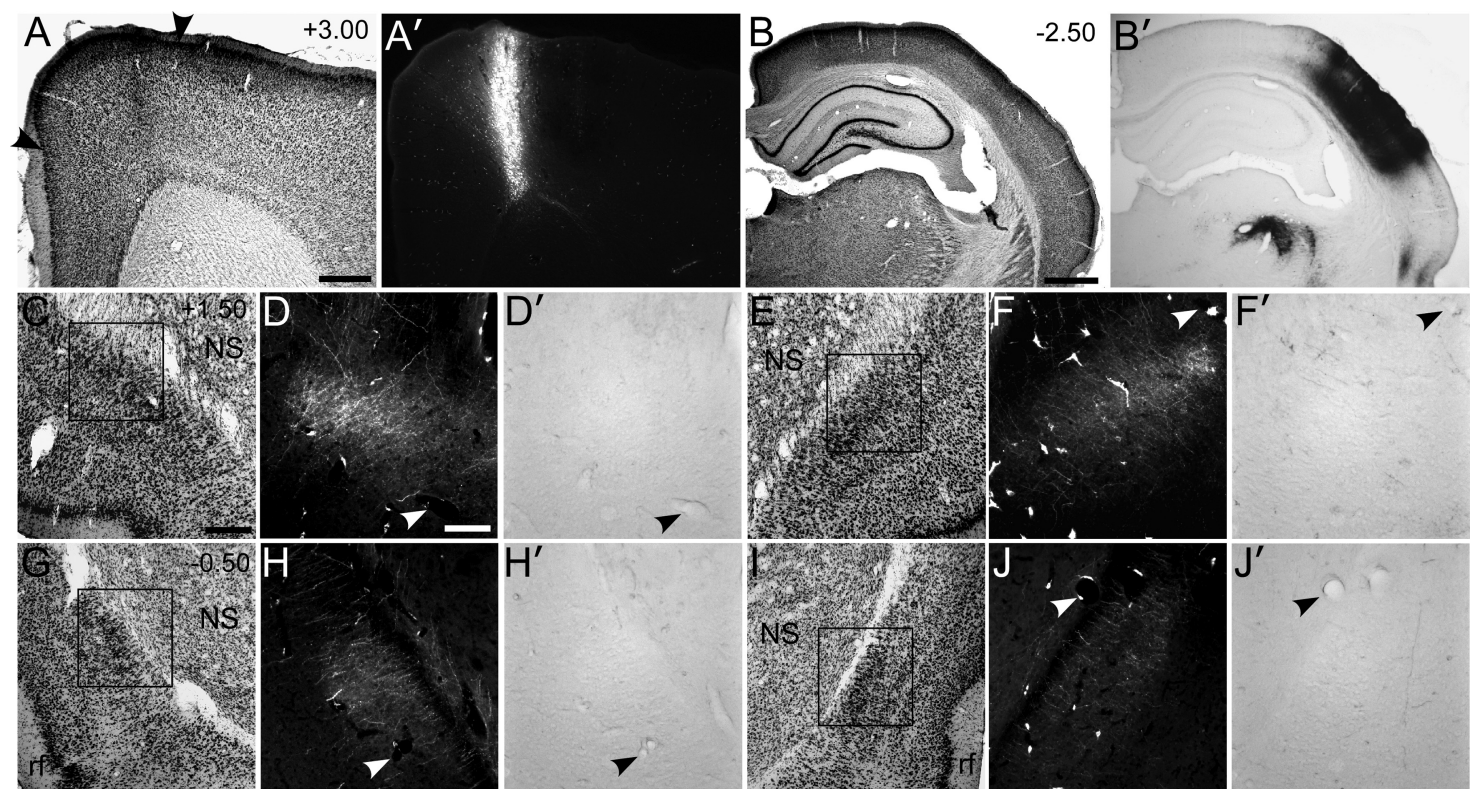

Figure 1. The claustrum receives projections from wMl but not from wSI. $\boldsymbol{A}, \boldsymbol{A}^{\prime}$, Nissl-stained section of wMl with adjacent section showing an FR injection in the right Agm (arrowheads). $\boldsymbol{B}, \boldsymbol{B}^{\prime}$, Nissl-stained section showing a BDA injection in the right wSI. Dense BDA labeling appears in VPM and POm of the thalamus. $C$, Nissl-stained section of the left claustrum $1.5 \mathrm{~mm}$ rostral to bregma. $D, D^{\prime}$, Dense FR-labeled but no BDA-labeled terminals in the claustrum. $\boldsymbol{E}$, Nissl-stained section of the right claustrum, $1.5 \mathrm{~mm}$ rostral to bregma. $\boldsymbol{F}, \boldsymbol{F}^{\prime}$, Weak FR-labeling in right claustrum, but no BDA labeling. $\mathbf{G}-J^{\prime}$, Similar patterns appeared in the left $\left(\mathbf{G}-\boldsymbol{H}^{\prime}\right)$ and right $\left(\boldsymbol{I}-\boldsymbol{J}^{\prime}\right)$ claustrum in sections $500 \mu \mathrm{m}$ caudal to bregma. Scale bars: $\boldsymbol{A}, 0.5 \mathrm{~mm} ; \boldsymbol{B}, 1 \mathrm{~mm} ; \boldsymbol{C}, 250 \mu \mathrm{m} ; \boldsymbol{D}, 100 \mu \mathrm{m}$. NS, Neostriatum; rf, rhinal fissure.

projects to both cortical regions. Finally, the anterograde tracers AF and FR were separately injected into corresponding parts of wMI in the two hemispheres, and these were coupled with an injection of the retrograde tracer FG into wSI $(n=2)$ to ascertain whether wMI projections from both hemispheres converge onto claustral neurons that project to wSI.

Our analysis of tracer labeling was limited to sections located within $2.5 \mathrm{~mm}$ of bregma that contained the striatum because these sections are associated with a claustrum-specific protein, Gng2 (Mathur et al., 2009). Nissl staining was used for one series of sections to visualize the claustrum as described previously (Paxinos and Watson, 2005; Smith and Alloway, 2010).

\section{Dual anterograde injections}

To ascertain whether the claustrum integrates whisker-related information from sensorimotor cortex, FR and BDA were injected into wMI and wSI, respectively, of the same hemisphere. Consistent with previous work (Brecht et al., 2004; Alloway et al., 2008, 2009; Smith and Alloway, 2010), FR injections in wMI were in agranular medial cortex (Agm), as illustrated in Figure 1. Tracer injections in wSI filled all layers of cortex and, as expected, revealed dense labeling in the thalamus, including the ventroposterior medial (VPM) and posterior medial (POm) nuclei (Hoffer and Alloway, 2001).

In both hemispheres, the claustrum received projections from wMI, but projections from wSI were not apparent. As described previously (Alloway et al., 2009; Smith and Alloway, 2010), labeled projections from wMI were densest in the contralateral claustrum. Whether projecting ipsilaterally or contralaterally, the labeled projections from MI coursed through the external capsule before terminating mainly in the anterior claustrum and, to a lesser degree, in more posterior parts of this structure.

By contrast, virtually no projections from wSI were seen in the claustrum of either hemisphere (Fig. 1). Despite the presence of extremely dense BDA-labeled axon terminals in the thalamus, especially in VPM and POm, BDA-labeled projections from SI were largely absent from the claustrum. Isolated fibers were occasionally observed in the claustrum located ipsilateral to the injection site, but these faintly labeled fibers did not contain varicosities indicative of en passant synapses. The lack of anterograde labeling in the claustrum following tracer injections in wSI is consistent with our previous report in which focal injections of retrograde tracers into the claustrum failed to produce retrogradely labeled neurons in SI cortex (Smith and Alloway, 2010).

\section{Dual retrograde injections}

Different retrograde tracers were injected into wMI and wSI to determine whether the same part of the claustrum projects to these cortical areas. An example of these injections in wMI and wSI appears in Figure 2. Labeled neurons produced by the wMI injection were seen in the claustrum of both hemispheres, but were more numerous in the ipsilateral claustrum, which contained $88.0 \%( \pm 3.8 \%)$ of all claustral labeling. Furthermore, retrograde labeling was strongest in the anterior claustrum and gradually declined in more posterior sections. Injections in wSI revealed labeling in the claustrum of both hemispheres, but also favored the ipsilateral hemisphere $(78.5 \pm 1.9 \%$ of total claustral labeling), as described previously (Li et al., 1986; Sloniewski et al., 1986).

The claustrum contained many labeled neurons projecting to both wSI and wMI. Claustral neurons projecting to these cortical regions were intermingled and included many double-labeled neurons, especially in the rostral half of this structure. The double-labeled neurons comprised a small portion of total claustral labeling $(4.0 \pm 0.5 \%)$, which approximately matches the small proportion of double-labeled neurons observed after tracer injections in other widely separated cortical regions (Minciacchi et al., 1985). As shown in Figure 2, confocal images of the claustrum in each hemisphere depict several double-labeled neurons in which blue soma contained scattered granules of FG. Some of 


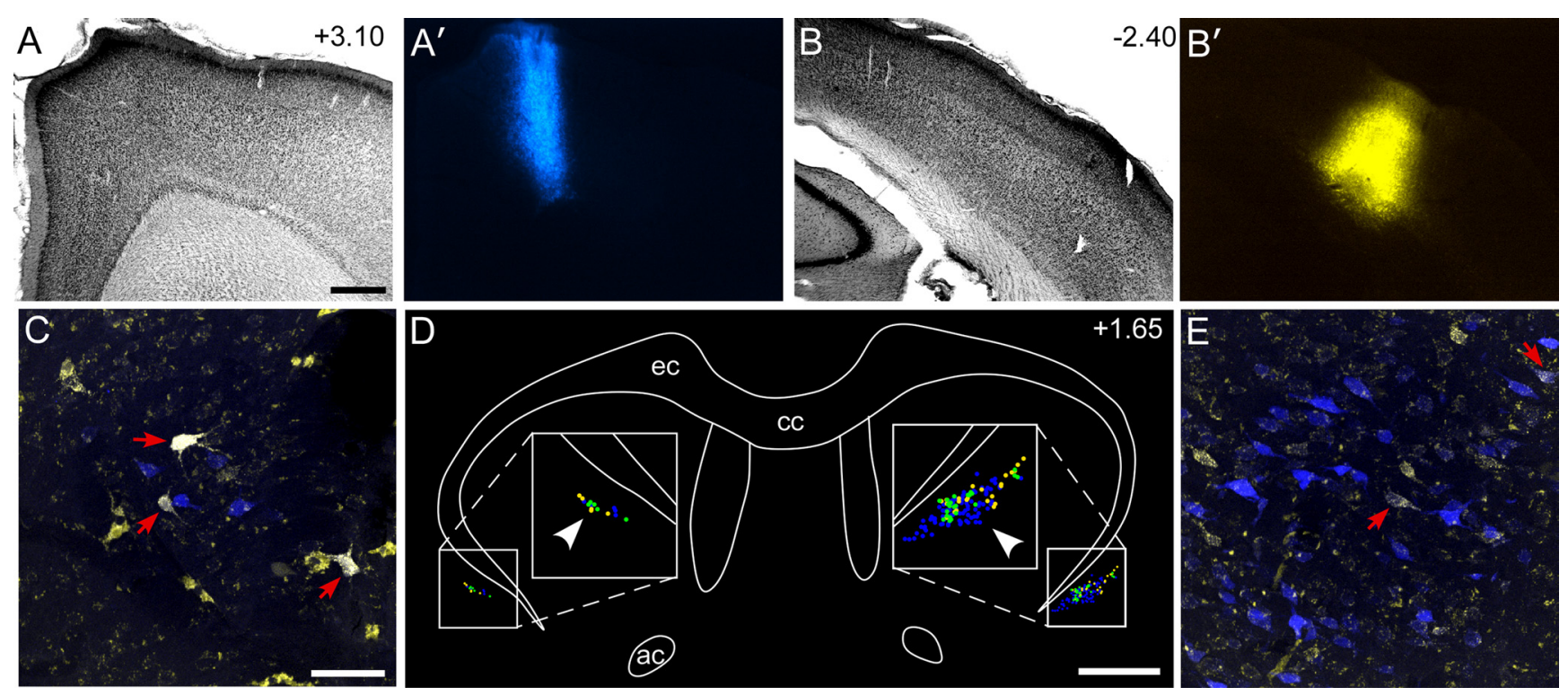

Figure 2. The claustrum projects to wMl and wSI. $A, A^{\prime}$, Injection of TB in the right wMI. $B, B^{\prime}$, Injection of FG in the right wSI. $D$, Reconstruction of retrogradely labeled neurons in the left and right claustrum showing intermingled TB (blue) and FG (gold) labeled neurons. Double-labeled neurons are shown in green. Arrowheads correspond to confocal images obtained from the claustrum in the left $(\boldsymbol{C})$ and right $(\boldsymbol{E})$ hemispheres. The most prominent double-labeled neurons are indicated with red arrows. Scale bar: $A, 0.5 \mathrm{~mm} ; \boldsymbol{C}, 50 \mu \mathrm{m} ; \boldsymbol{D}, 1 \mathrm{~mm}$. ac, Anterior commissure; $\boldsymbol{C}$, corpus callosum; ec, external capsule.
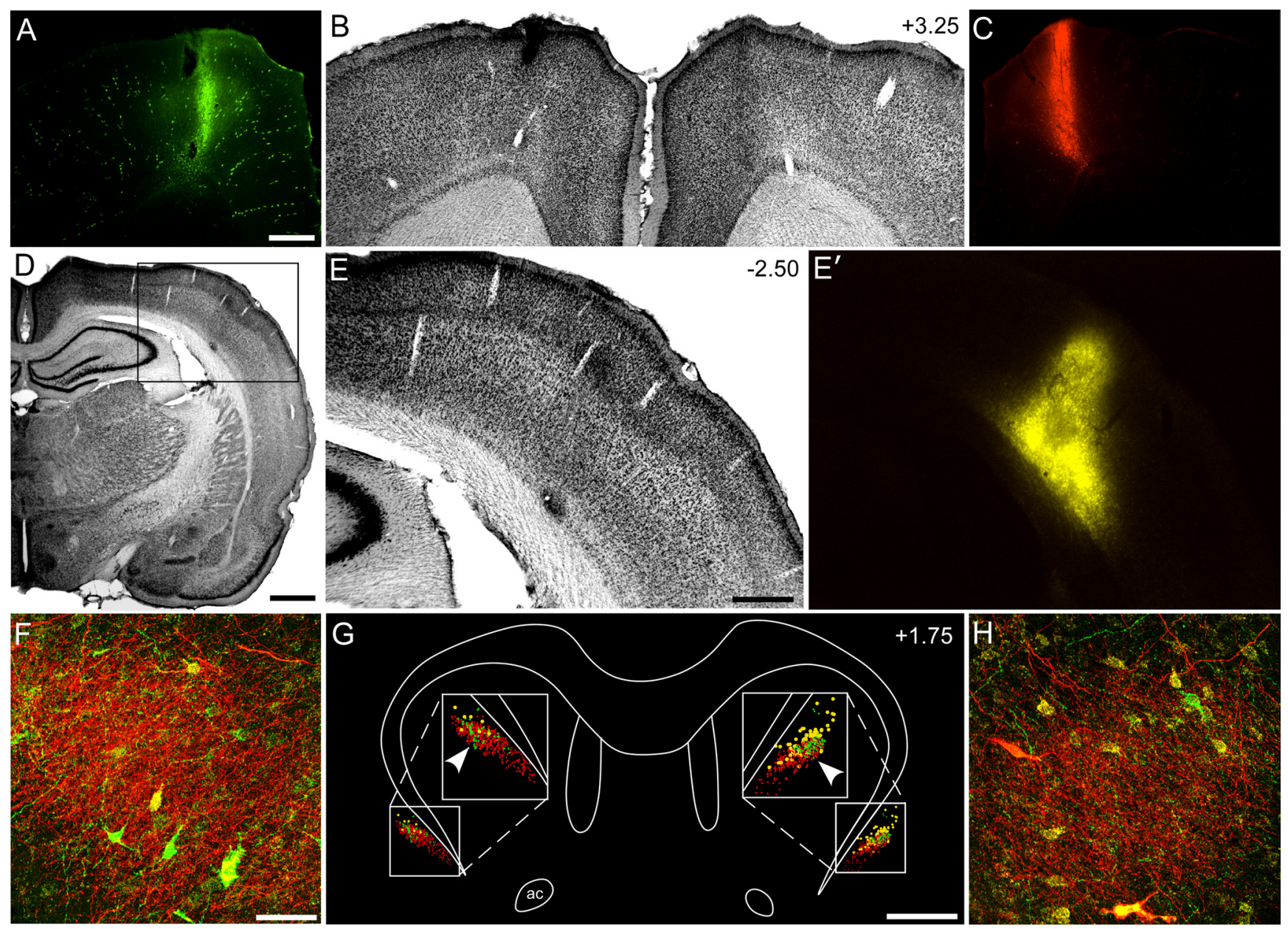

Figure 3. The same claustral region receives wMl inputs from both hemispheres and projects to the ipsilateral wSI. $A-C, A F$ and FR injections in wMl of the left and right hemispheres, respectively. $D-E^{\prime}, F G$ injection in the right wSI. G, Bilateral reconstructions of tracer labeling in the claustrum. AF- and FR-labeled terminals shown as green and red dots, respectively; FG-labeled neurons shown as gold circles. $\boldsymbol{F}, \boldsymbol{H}$, Arrowheads indicate sites for confocal images from left $(\boldsymbol{F})$ and right $(\boldsymbol{H})$ hemispheres. Dense AF and FR terminal labeling surrounds FG-labeled neurons on both sides. Scale bars: $\boldsymbol{A}, \boldsymbol{E}, 0.5 \mathrm{~mm} ; \boldsymbol{D}, 1 \mathrm{~mm} ; \boldsymbol{F}, 50 \mu \mathrm{m} ; \boldsymbol{G}, 1 \mathrm{~mm}$. ac, Anterior commissure. 
these double-labeled neurons were exceptionally bright and had very large soma (Fig. $2 C$ ). This suggests that these neurons received more transported tracer than the other labeled neurons, possibly because the largest neurons have the most extensive terminal arbors.

\section{Triple tracer injections}

In two experiments, we combined anterograde and retrograde tracer injections to determine whether the wMI and wSI regions are interhemispherically connected by a cortico-claustro-cortical circuit. The anterograde tracers $\mathrm{AF}$ and $\mathrm{FR}$ were injected into wMI of the left and right hemispheres (Fig. $3 A-C$ ), respectively, with injections of the retrograde tracer FG in wSI of the right hemisphere (Fig. 3D-E'). The bilateral injections of anterograde tracers in wMI produced bilateral claustral labeling in which labeled terminals were denser in the hemisphere contralateral to the injection site. Axon terminals labeled by FR were more prominent than AF-labeled terminals, and this obscured visualization of the full extent of AF-labeling when tissue was viewed with conventional microscopy. Confocal microscopy, however, revealed both FR- and AF-labeled terminals in overlapping parts of the claustrum of each hemisphere, especially in the anterior and central portions of this structure. These results are consistent with our previous report showing that MI projects mainly to the contralateral claustrum, and that nearly $10 \%$ of the innervated territory also receives projections from the ipsilateral MI whisker region (Alloway et al., 2009). In addition, labeled projections from the MI region in each hemisphere overlapped with most, but not all (Fig. $3 G$ ), of the claustral neurons that were retrogradely labeled by FG tracer injections in wSI. This suggests the existence of a cortico-claustral-cortical circuit in which information received from the wMI of each hemisphere is transmitted ipsilaterally to the wSI region.

\section{Discussion}

This neuroanatomical tracing study revealed three major findings. First, injections of different anterograde tracers in wMI and wSI revealed that the claustrum does not integrate information from these sensorimotor regions; in fact, wSI does not project to the claustrum of either hemisphere. Second, injections of retrograde tracers in wMI and wSI revealed overlapping populations of labeled neurons with many double-labeled neurons. This indicates that the claustrum transmits information to both cortical regions, and suggests that the rodent claustrum has a role in sensorimotor coordination. Finally, anterograde tracer injections in wMI of each hemisphere, coupled with a single retrograde injection in wSI, revealed that wMI projects to the claustral regions that project to wSI. This demonstrates an interhemispheric cortico-claustral-cortical circuit that could supplement the ipsilateral corticocortical projections from MI to SI in each hemisphere.

Many studies report that cortex is reciprocally connected with the claustrum, but most of these findings are in cats (Olson and Graybiel, 1980; Edelstein and Denaro, 2004). In contrast, tracing studies in rodents and primates indicate that cortical areas 17 and 18 do not innervate the claustrum, but they do receive projections from the claustrum (Carey and Neal, 1985; Sherk, 1986). The present study indicates that the claustrum projects to wSI cortex, but this cortical region does not reciprocate. Hence, the somatosensory pattern in rats is similar to the nonreciprocal pattern described for the visual areas in rats and primates, but differs from the reciprocal pattern seen in cats. This is significant because rodents and primates are phylogenetically close, both be- longing to the Euarchontoglires superorder, whereas cats belong to the Laurasiatheria superorder (Murphy et al., 2004). These data suggest evolutionary differences in the connectivity between the claustrum and cortex, which may have implications for understanding claustral functions.

Another theory of claustral function centers on sensorimotor integration (Pearson et al., 1982; Edelstein and Denaro, 2004). Our present findings indicate that the rat claustrum does not integrate inputs from SI and MI. This is consistent with previous results from our lab showing that retrograde tracer injections into the claustrum revealed dense neuronal labeling in MI but failed to reveal any labeled neurons in SI or in any of the surrounding cortical areas (Smith and Alloway, 2010). Nonetheless, the present study suggests a role for the claustrum in transmitting information to both somatosensory and motor cortical areas. Simultaneous neuronal recordings in wSI and wMI have revealed correlated discharges in these regions during spontaneous and stimulus-evoked activity (Chakrabarti et al., 2008). Divergent projections from the claustrum could represent one of many routes for coordinating the flow of motor-related information to both of these cortical regions.

In addition to motor control, Agm (wMI) has been implicated as having a role in selective attention and memory-guided orienting (Reep and Corwin, 2009; Erlich et al., 2011). This view is based on data showing that Agm receives input from multiple somesthetic and association cortical areas involved in spatial attention and memory (Reep et al., 1987; Colechio and Alloway, 2009). This is significant when considered with our data showing that Agm innervates the claustrum. This pattern of neural connectivity suggests that Agm transmits sensorimotor-related information to the claustrum that is important for coordinating motor behaviors involved in redirecting spatial attention.

\section{References}

Alloway KD, Mutic JJ, Hoover JE (1998) Divergent corticostriatal projections from a single cortical column in the somatosensory cortex of rats. Brain Res 785:341-346.

Alloway KD, Olson ML, Smith JB (2008) Contralateral corticothalamic projections from MI whisker cortex: potential route for modulating hemispheric interactions. J Comp Neurol 510:100-116.

Alloway KD, Smith JB, Beauchemin KJ, Olson ML (2009) Bilateral projections from rat MI whisker cortex to the neostriatum, thalamus, and claustrum: forebrain circuits for modulating whisking behavior. J Comp Neurol 515:548-564.

Brecht M, Krauss A, Muhammad S, Sinai-Esfahani L, Bellanca S, Margrie TW (2004) Organization of rat vibrissa motor cortex and adjacent areas according to cytoarchitectonics, microstimulation, and intracellular stimulation of identified cells. J Comp Neurol 479:360-373.

Carey RG, Neal TL (1985) The rat claustrum: afferent and efferent connections with visual cortex. Brain Res 329:185-193.

Chakrabarti S, Zhang M, Alloway KD (2008) MI neuronal responses to peripheral whisker stimulation: relationship to neuronal activity in SI barrels and septa. J Neurophysiol 100:50-63.

Colechio EM, Alloway KD (2009) Differential topography of the bilateral cortical projections to the whisker and forepaw regions in rat motor cortex. Brain Struct Funct 213:423-439.

Cortimiglia R, Crescimanno G, Salerno MT, Amato G (1991) The role of the claustrum in the bilateral control of frontal oculomotor neurons in the cat. Exp Brain Res 84:471-477.

Crick FC, Koch C (2005) What is the function of the claustrum? Philos Trans R Soc Lond B Biol Sci 360:1271-1279.

Edelstein LR, Denaro FJ (2004) The claustrum: a historical review of its anatomy, physiology, cytochemistry and functional significance. Cell Mol Biol (Noisy-le-grand) 50:675-702.

Erlich JC, Bialek M, Brody CD (2011) A cortical substrate for memoryguided orienting in the rat. Neuron 72:330-343.

Haiss F, Schwarz C (2005) Spatial segregation of different modes of move- 
ment control in the whisker representation of rat primary motor cortex. J Neurosci 25:1579-1587.

Hall RD, Lindholm LE (1974) Organization of motor and somatosensory neocortex in the albino rat. Brain Res 66:23-38.

Hoffer ZS, Alloway KD (2001) Organization of corticostriatal projections from the vibrissal representations in the primary motor and somatosensory cortical areas of rodents. J Comp Neurol 439:87-103.

Hoffer ZS, Hoover JE, Alloway KD (2003) Sensorimotor corticocortical projections from rat barrel cortex have an anisotropic organization that facilitates integration of inputs from whiskers in the same row. J Comp Neurol 466:525-544.

Kincaid AE, Wilson CJ (1996) Corticostriatal innervation of the patch and matrix in the rat neostriatum. J Comp Neurol 374:578-592.

Land PW, Simons DJ (1985) Cytochrome oxidase staining in the rat SmI barrel cortex. J Comp Neurol 238:225-235.

Li ZK, Takada M, Hattori T (1986) Topographic organization and collateralization of claustrocortical projections in the rat. Brain Res Bull 17:529-532.

Mathur BN, Caprioli RM, Deutch AY (2009) Proteomic analysis illuminates a novel structural definition of the claustrum and insula. Cereb Cortex 19:2372-2379.

Minciacchi D, Molinari M, Bentivoglio M, Macchi G (1985) The organization of the ipsi- and contralateral claustrocortical system in rat with notes on the bilateral claustrocortical projections in cat. Neuroscience 16:557-576.

Mitchinson B, Martin CJ, Grant RA, Prescott TJ (2007) Feedback control in active sensing: rat exploratory whisking is modulated by environmental contact. Proc Biol Sci 274:1035-1041.

Murphy WJ, Pevzner PA, O’Brien SJ (2004) Mammalian phylogenomics comes of age. Trends Genet 20:631-639.
Olson CR, Graybiel AM (1980) Sensory maps in the claustrum of the cat. Nature 288:479-481.

Paxinos G, Watson C (2005) The rat brain in stereotaxic coordinates, 6th edition. New York: Academic.

Pearson RC, Brodal P, Gatter KC, Powell TP (1982) The organization of the connections between the cortex and the claustrum in the monkey. Brain Res 234:435-441.

Reep RL, Corwin JV (2009) Posterior parietal cortex as part of a neural network for directed attention in rats. Neurobiol Learn Mem 91:104-113.

Reep RL, Corwin JV, Hashimoto A, Watson RT (1987) Efferent connections of the rostral portion of medial agranular cortex in rats. Brain Res Bull 19:203-221.

Remedios R, Logothetis NK, Kayser C (2010) Unimodal responses prevail within the multisensory claustrum. J Neurosci 30:12902-12907.

Sanderson KJ, Welker W, Shambes GM (1984) Reevaluation of motor cortex and of sensorimotor overlap in cerebral cortex of albino rats. Brain Res 292:251-260.

Sherk H (1986) The claustrum and the cerebral cortex. In: Cerebral cortex: sensory-motor areas and aspects of cortical connectivity (Jones EG, Peters A, eds.), pp 467-499. New York: Plenum.

Sloniewski P, Usunoff KG, Pilgrim C (1986) Retrograde transport of fluorescent tracers reveals extensive ipsi- and contralateral claustrocortical connections in the rat. J Comp Neurol 246:467-477.

Smith JB, Alloway KD (2010) Functional specificity of claustrum connections in the rat: interhemispheric communication between specific parts of motor cortex. J Neurosci 30:16832-16844.

Towal RB, Hartmann MJ (2006) Right-left asymmetries in the whisking behavior of rats anticipate head movements. J Neurosci 26:8838-8846.

Voigt T, De Lima AD, Beckmann M (1993) Synaptophysin immunohistochemiostry reveals inside-out pattern of early synaptogenesis in ferret cerebral cortex. J Comp Neurol 330:48-64. 